\title{
FLOW PATTERN IDENTIFICATION IN A RECTANGULAR TANK COMPRISING STIRRER IN AN OFF-CENTER ORIENTATION
}

\author{
G. Ligus*, M. Wasilewski**
}

\begin{abstract}
In this paper the results of experimental research of flow pattern identification during mixing process using a stirrer in an off-center orientation in a rectangular tank were presented. The research applied Digital Particle Image Velocimetry (DPIV) technique. The velocity vector fields with regard to the stirrer orientation and rotational speed of the mixing process were determined and they were subsequently applied for identification and assessment of liquid flow pattern in a tank. Also the assessment of flow pattern by statistical non-uniform coefficient was introduced. The relation between the rotational speed of the stirrer and stirrer orientation for mixing performance were determined. The study demonstrated some lack of analogy in certain areas of liquid behavior during the mixing process in the tank with rectangular crosssection and circular cross-section.
\end{abstract}

Keywords: mixing, off-center, rectangular tank, stirrer, DPIV

\section{Introduction}

Liquid mixing are widely used processes in the majority of industry. On that account an assessment of flow behaviour in a stirred tank is the subject of numerous experimental and numerical studies (Virdung et al., 2008; Blais et al., 2016; Sharp et al., 2001; Long and Nong, 2017; Malik and Pakzad, 2018; Wang et al., 2016; Lamotte et al., 2018). The change in the quality of the mixing process can be achieved by changing many parameters for example: type and number of stirrer, rotation speed of stirrer, geometry of the tank or an orientation of the stirrer in the tank (Kresta et al., 2016). In the aspect of modifying the spatial orientation of the stirrer, two types of solutions are practiced. Change of the angle of stirrer rotation and change of the stirrer orientation relative to the center of the tank (Moss, 2004). It should be pointed out that the flow patterns of liquid mixing in a such modified geometry are complex in description. In this situation, the visualization techniques could be very helpful. One of the possible ways to reach information basis on the visualization is Digital Particles Image Velocimetry (DPIV) method. The first scientists who introduced DPIV method to the investigation of nature of the mixing process in stirred tanks were Ward (Ward, 1995). Due to the wide range of applications, the DPIV method has gained importance in the study of the mixing process. The aim of presented research is an attempt to assessment of liquid flow patterns in a rectangular tank comprising stirrer in an off-center orientation and compared with the flow patterns obtained with the stirrer in the central orientation. To achieve this aim, the paper contains correlation of visual identification of flow pattern with statistical analysis of nonuniform coefficient of mixing process.

\section{Methods}

Experimental research were carried out in a rectangular tank with the dimensions of $0.23 \times 0.23 \times 0.23 \mathrm{~m}$. Three stirrer orientations were adopted: two off-central orientations and central orientation for comparative analysis. Application of DPIV method for liquid flow pattern identification required use of

\footnotetext{
Grzegorz Ligus, PhD.: Faculty of Mechanical Engineering, Opole University of Technology, 76 Proszkowska St., 45-758 Opole, Poland, g.ligus@po.opole.pl

** $\quad$ Marek Wasilewski, PhD.: Faculty of Production Engineering and Logistics, Opole University of Technology, 76 Proszkowska St., 45-758 Opole, Poland, m.wasilewski@po.opole.pl
} 
Nd:YAG laser synchronized with a CCD camera. Laser sheet generated by optic elements illuminated the tank in horizontal plane in half of the height of the liquid level. The tank was filled with water and contained seeding particles (PSP20) made of polyamide material. Mixture of water with seeding particles occupied the tank part of up to $0.15 \mathrm{~m}$ in height. The parameters of the seeding particles (particle diameter at the level of $20 \mu \mathrm{m}$ and density equal to $1.03 \mathrm{~g} / \mathrm{cm}^{3}$ ) make them almost perfectly neutrally buoyant in water (Ardian and Westerweel, 2011). The experiment used a magnetic stirring technique. Inside the tank, there is a cylindrical magnetic stir bar with the length of $0.045 \mathrm{~m}$ and the diameter of 0.01 $\mathrm{m}$. The ends of the cylindrical bar are rounded. The experiment was performed for four rotational speeds, equal to 250, 500, 750 and $1000 \mathrm{rpm}$. The diagram of the measurement setup and stirrer orientation in the tank are presented in Fig. 1.

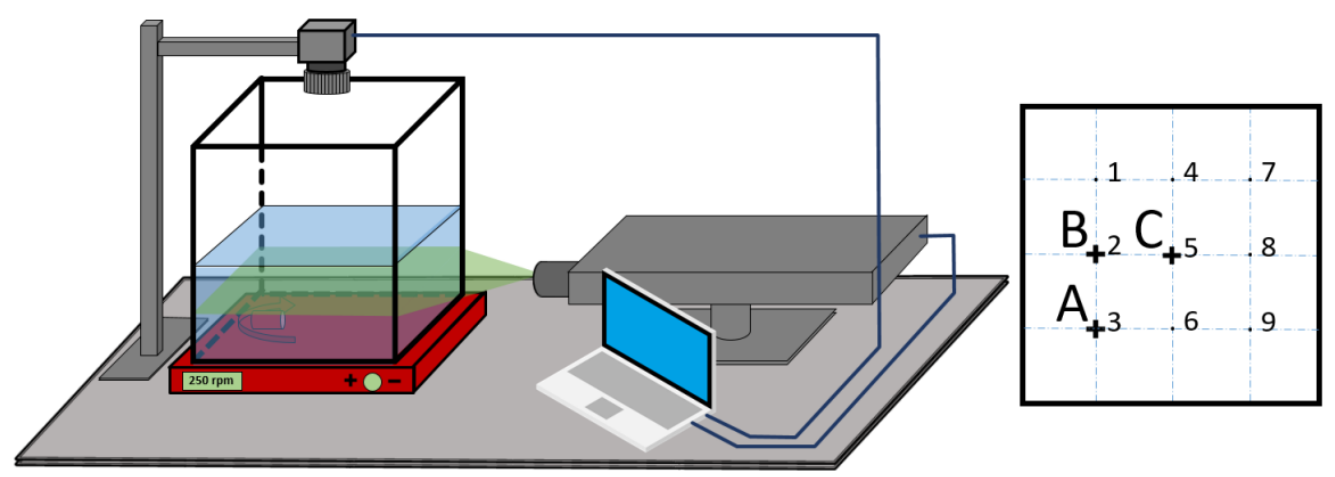

Fig. 1: Experimental stand with measurement area description (A, B, $C$-stirrer orientation, 1-9 measurement points of velocity variability analysis on the laser sheet).

The adaptive correlation method calculates velocity vectors with an initial interrogation area (IA) of the size $\mathrm{N}$ in the size of the final IA. Applied calculation algorithm uses the intermediary results as information for the next IA of smaller size, until the final IA size is reached. Velocity vectors are estimated from mean particle displacement inside interrogation areas (IA) according to the following equations:

$$
D\left(\vec{X} ; t_{0}, t_{+1}\right) \approx \int_{t_{0}}^{t_{+1}} u[\vec{X}(t), t] d t
$$

where $\mathrm{D}$ is the displacement and ' $\mathrm{u}$ ' is the velocity, is the main formula used to calculate velocity vectors. For detection and substituting false vector the Universal Outlier Detection (UOD) was applied. UOD based on a normalized median test using the surrounding vectors (Westerweel and Scarano, 2005). The normalized vector residuals can be calculated as:

$$
r_{0}=\frac{\left|U_{0}-U_{m}\right|}{r_{m}+\varepsilon}
$$

where $\mathrm{U}_{0}$ is the displacement vector, $\mathrm{U}_{\mathrm{m}}$ is the median vector calculated using the neighbourhood vectors $\left\{\mathrm{U}_{1}, \mathrm{U}_{2}, \ldots, \mathrm{U}_{\mathrm{M}} \times \mathrm{N-1}\right], \mathrm{r}_{\mathrm{m}}$ is the median residual calculated using the neighbourhood residuals $\left\{\mathrm{r}_{1}, \mathrm{r}_{2}, \ldots, \mathrm{r}_{\mathrm{m} \times \mathrm{N}-1}\right\}$ where $\mathrm{r}_{\mathrm{i}}=\left|\mathrm{U}_{\mathrm{i}}-\mathrm{U}_{\mathrm{m}}\right|$ for $\{\mathrm{i}=1, \ldots, \mathrm{M} \times \mathrm{N}-1\}$ and $\varepsilon$ is the minimum normalization level. If the normalized vector residual is above the detection threshold, the displacement vector can be substituted by its median vector, otherwise the vector is left unchanged (Dantec Dynamics, 2015). For assesement of velocity varability non-uniform coefficient of mixing $k_{v}$ was introduced. It was assumed that the local decrease in liquid flow velocity is caused by the influence of neighbouring liquid streams with other component velocities $U$ and $V$. Therefore, the calculation of the local velocity variation allows to determine the intensity of mixing. For this purpose, from all 200 pairs of images at all measurement points extraction of instantaneous local velocity were made and subjected to statistical evaluation according to equation (3). A similar procedure was used in ( $\mathrm{Li}$ at al., 2018). The higher $\mathrm{k}_{\mathrm{v}}$ coefficient, the more non-uniform flow of liquid at the measuring point. As a consequence, high mixing efficiency should be characterized by high $\mathrm{k}_{\mathrm{v}}$ coefficients.

$$
k_{V}=\frac{\sqrt{\frac{\sum_{i=1}^{n}\left(V_{i}-\bar{V}\right)^{2}}{n}}}{\frac{\sum_{i=1}^{n} V_{i}}{n}}
$$

where: $V_{\mathrm{i}}$ - instantaneous velocity, $\bar{V}$ - mean velocity, n - number of measurements. 


\section{Results}

The identification of the flow pattern using non-uniform coefficient of mixing $\mathrm{k}_{\mathrm{v}}$ based an analysis of the velocity vector field (Fig. 2.). For all series of presented research, velocity vector fields were determined with histograms of velocity and extracted local velocities at all measurement points. Each designated velocity vector is the resultant of two components in direction $U$ and V. For the case shown in Fig. 2, three vortex patterns were identified. The main vortex, which is the direct effect of the rotary motion of the stirrer at point $\mathrm{A}$, and two derivative vortices located on the symmetry axis of the tank crossing points $\mathrm{A}$ and $\mathrm{C}$. The number of border areas between vortices with opposite directions of rotation due to the liquid streams intersecting in them, may constitute a parameter describing the mixing process qualitatively. The number of this border areas is connected with global number of the vortices and also with the size of the vortices. This two parameters vary both due to changes of rotational speed and orientation of the stirrer. Quantity and size of the vortex patterns increases with the rotational speed. However, it was noticed that the mixing process performed in both off-center stirrer orientations, generates flow patterns with a more uniform distribution compared to the central orientation. In view of the above, it was found that for rectangular tanks the displacement of the stirrer to the off-center orientation does not give the effect in the form of an increase in mixing performance. This regularity is clearly represented in the values of the $\mathrm{k}_{\mathrm{v}}$ coefficient shown in Fig. 3. Regardless of the rotational speed, the highest non-uniform coefficients have been achieved for the central orientation of the stirrer. In the aspect of the stirrer orientation, there is no analogy in flow patterns of the mixing process occurring in tanks with a rectangular cross-section and circular cross-section.

a)
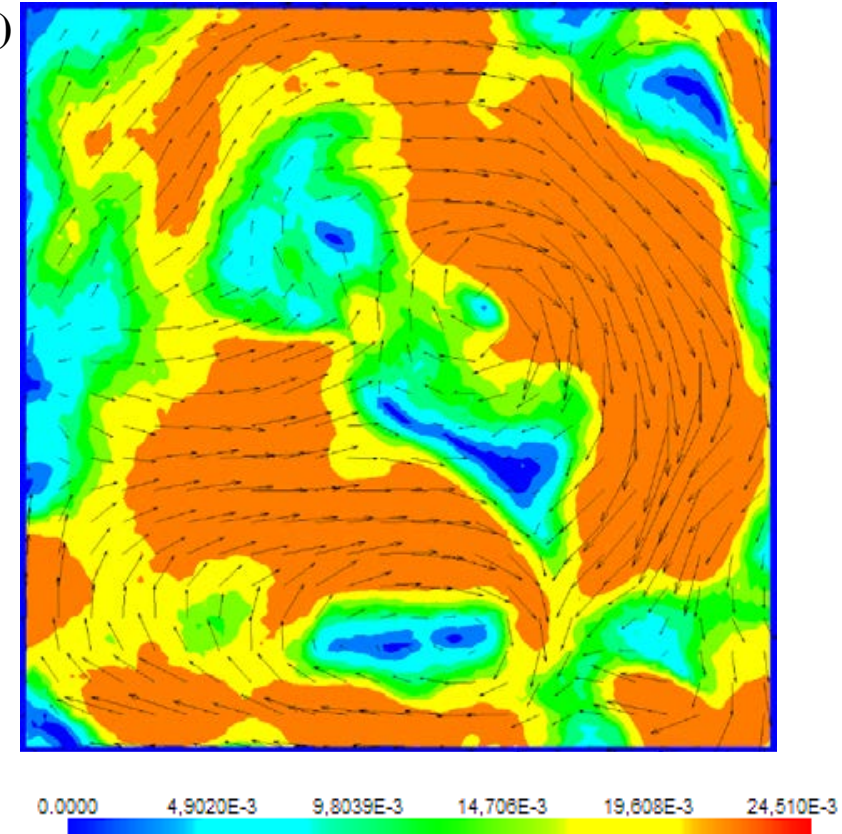

b)
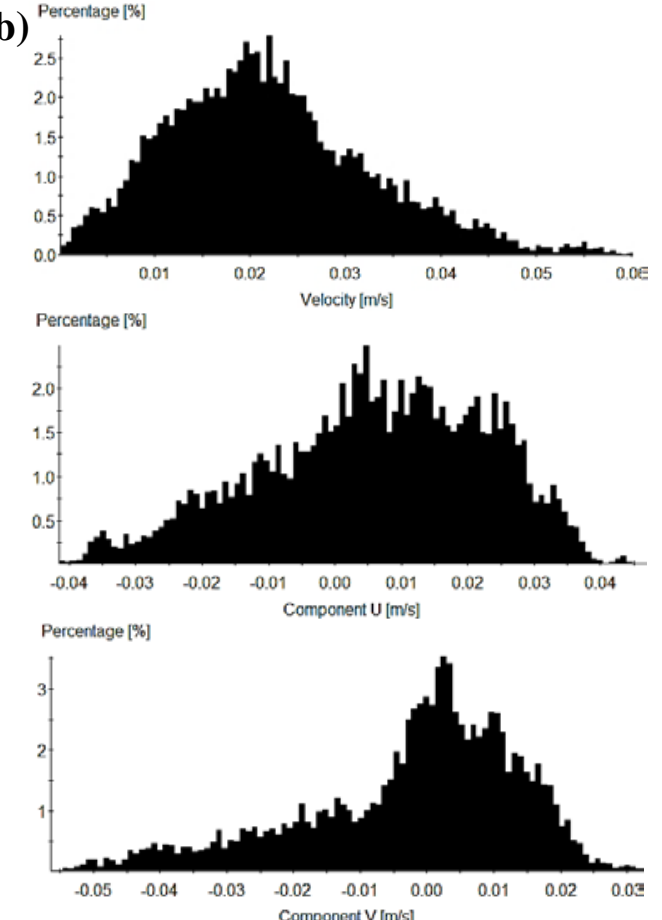

c)

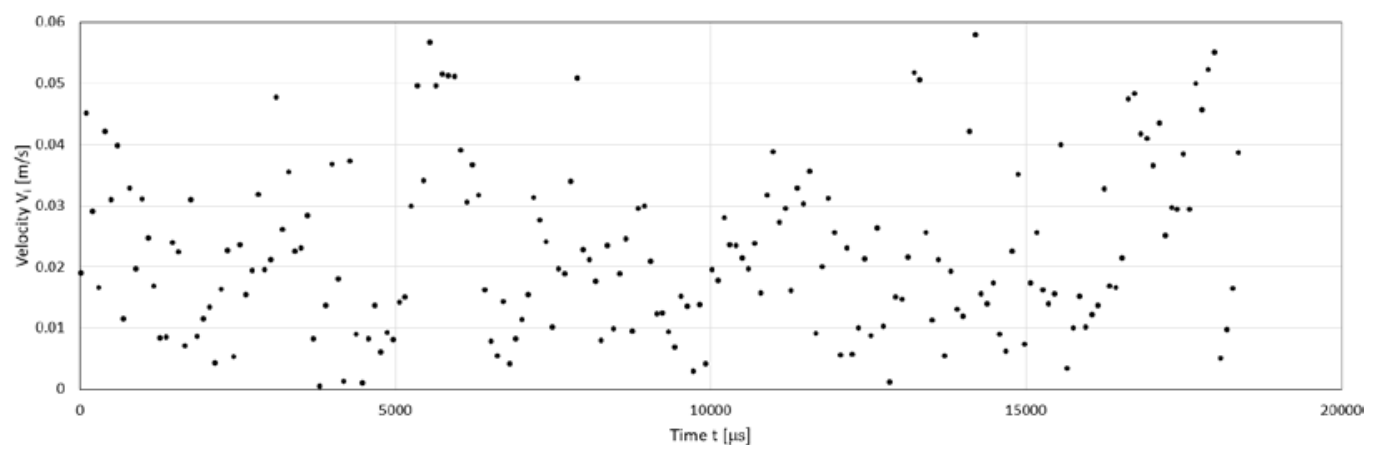

Fig. 2: Exemplary results for stirrer orientation at point A and rotational speed equal to $500 \mathrm{rpm}$ : a) velocity vector field (value of velocity in $\mathrm{m} / \mathrm{s}$ ), b) global histogram of velocity with decomposition on components $U$ and $V$, c) extracted instantaneous velocity at point 4. 


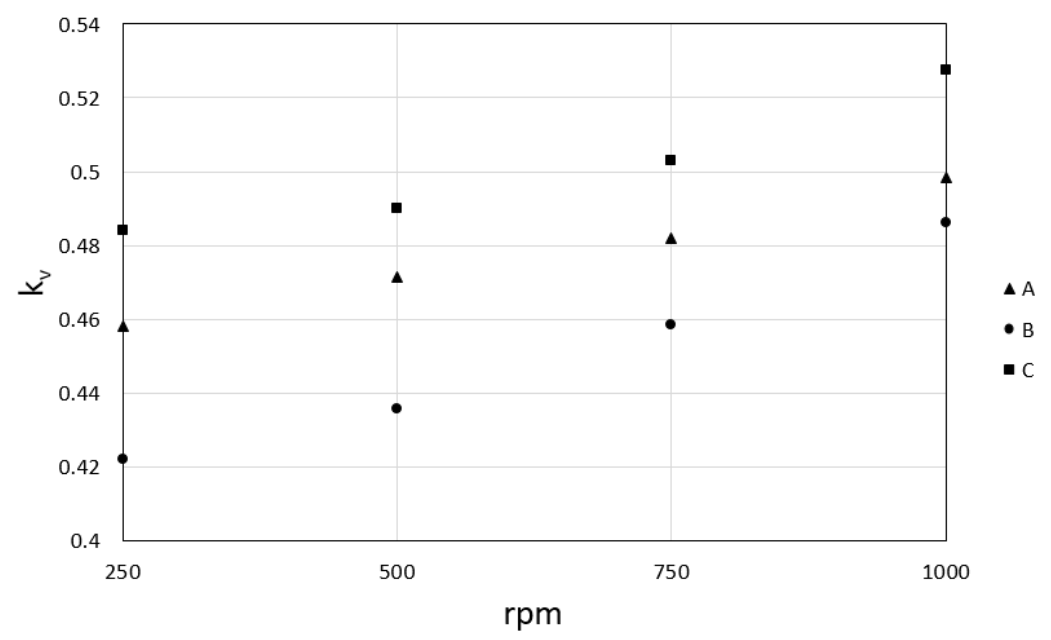

Fig. 3: Global non-uniform coefficient of mixing $k_{v}$ in rectangular tank ( $A, B, C$ - stirrer orientation)

\section{Conclusions}

On the basis of the measurements and analysis the following conclusions were formulated:

- DPIV method is a useful tool for flow pattern identification during mixing processes and makes possible to describe flow patterns by non-uniform coefficients of mixing,

- The number and size of the vortex patterns increases with the rotational speed,

- Shaping the flow patterns under influence of stirrer displacement to off-center orientation does not occur in the same way in a tank with rectangular cross-section and tank with circular cross-section,

- In a rectangular tank comprising stirrer in a both tested off-center orientations, compared to the central orientation, no increase in the mixing performance has been observed.

\section{References}

Adrian R. J., Westerweel J. (2011) Particle Image Velocymetry, Cambridge University Press, New York.

Blais B., Lassaigne M., Goniva C., Fradette L., Bertrand F. (2016) Development of an unresolved CFD-DEM model for the flow of viscous suspensions and its application to solid-liquid mixing. Journal of Computational Physics, 318, pp. 201-221.

Dantec Dynamics. (2015) Dynamic Studio 2015a, User’s guide, Skovlunde.

Kresta S. M., Etchells A. W., Dickey D. S., Atiemo-Obeng V. A. (2016) Advances in Industrial Mixing. John Wiley \& Sons, Hoboken.

Lamotte A., Delafosse A., Calvo S., Toye D. (2018) Analysis of PIV measurements using modal decomposition techniques, POD and DMD, to study flow structures and their dynamics within a stirred-tank reactor. Chemical Engineering Science, 178, pp. 348-366.

Li A., Ren T., Yang Ch., Xiong J., Tao P. (2018) Numerical simulation, PIV measurements and analysis of air movement influenced by nozzle jets and heat sources in underground generator hall, Building and Environment, 131, pp. 16-31.

Long F., Nong X. (2017) Study on laminar flow containing fibre particles in a stirred tank using DPIV. Powder Technology, 320, pp. 420-427.

Malik D., Pakzad L. (2018) Experimental investigation on an aerated mixing vessel through electrical resistance tomography (ERT) and response surface methodology (RSM). Chemical Engineering Research and Design 129, pp. 327-343.

Moss, D. R. (2004) Pressure Vessel Design Manual. Elsevier, Amsterdam.

Scarano F. (2005) Universal outlier detection for PIV data. Experiments in fluids 39, 6, pp. 1096-1100.

Sharp K. V., Arian R. J. (2001) PIV study of small scale row structure around a Rushton impeller turbine. American Institute of Chemical Engineers Journal, 47, 4, pp. 766-778.

Virdung T., Rasmuson A. (2008) Solid-liquid flow at dilute concentrations in an axially stirred vessel investigated using Particle Image Velocimetry. Chemical Engineering Communications, vol. 195, 1, pp. 18-34.

Wang S., Stewart R., Metcalfe G. (2016) Visualization of the trapping of inertial particles in a laminar mixing tank. Chemical Engineering Science, 143, pp. 99-104.

Ward R.W. (1995) A DPIV Investigation of Flow Pattern Instabilities of Axial-Flow Impellers, University of Dayton, Dayton. 